\title{
A COMPARATIVE STUDY OF ANKLE-ARM PRESSURE INDEX IN CORONARY ARTERIAL DISEASE PATIENTS AND NORMAL POPULATION - A SOUTH INDIAN TERTIARY CARE CENTRE EXPERIENCE
}

\author{
Senathipathi Vengojayaprasad ${ }^{1}$, Alagianambi Shanmugam², Stephen Charles Bronson 3 , Duraisamy Venkatesh ${ }^{4}$, \\ Thayanithi Jayapackiam 5 , Duvuru Shantharam 6 \\ ${ }^{1}$ Associate Professor, Department of Diabetology, Coimbatore Medical College, Coimbatore. \\ ${ }^{2}$ Associate Professor, Institute of Diabetology, Stanley Medical College, Chennai. \\ 3Senior Resident, Institute of Diabetology, Stanley Medical College, Chennai. \\ ${ }^{4}$ Senior Resident, Department of Diabetology, Coimbatore Medical College, Coimbatore. \\ ${ }^{5}$ Assistant Professor, Institute of Diabetology, Stanley Medical College, Chennai. \\ ${ }^{6}$ Director and Professor, Institute of Diabetology, Stanley Medical College, Chennai.
}

ABSTRACT

\section{BACKGROUND}

Clinical manifestation of peripheral arterial disease confirmed by reduced ankle-brachial pressure index (ABPI) ranges from the common asymptomatic disease to the less prevalent intermittent claudication, to the relatively rare critical limb ischaemia with rest-pain, ulceration or gangrene. Even if peripheral arterial disease does not cause typical claudication, it reduces walking speed and walking endurance.(1) Regardless of the clinical symptoms, reduced ABPI is a sign of haemodynamic disturbance in the arterial supply of the lower limbs, which is strongly associated with atherosclerosis in the coronary and carotid territories.(2) Thus, peripheral arterial disease is associated with increased mortality due to myocardial infarction and ischaemic stroke. Several studies described 2-3-fold greater mortality in patients with peripheral arterial disease in comparison with age-matched controls with normal ABPI; patients with peripheral arterial disease had a 5-year mortality of about 30\%.(3-8) Increasing severity of peripheral arterial disease, expressed as diminishing ABPI, progressively reduces survival.(5-8) Most previously studied cohorts were either patients referred for non-invasive vascular testing(5) or patients with known risk factors such as hypertension(7) or hyperlipidaemia.(3)

Objectives - To study the prevalence of low ankle brachial pressure index in people with coronary arterial disease when compared with general population and to find out whether ABPI is a significant individual risk factor for coronary arterial disease.

\section{MATERIALS AND METHODS}

ABPI was compared between 100 patients with clinical cardiovascular disease versus 100 controls taken from patients attending Coimbatore Medical College and general population of Coimbatore respectively. ABPI $<0.9$ was taken as cut-off as it has a sensitivity of $90 \%$ in detecting peripheral vascular disease, which may also reflect similar pathology in other vascular bed, like coronary arteries. Prevalence of ABPI less than 0.9 was compared and analysed between the two study populations.

\section{RESULTS}

Of the 100 patients with coronary arterial disease, $34 \%$ had an ABPI value below 0.9 . ABPI in normal range of 0.9 to 1.2 was found in $58 \%$ of total. Another $8 \%$ had ABPI in higher range, which is usually associated with calcification of the vessel, which again is a risk for coronary arterial disease.

\section{CONCLUSION}

The study can be concluded by the remark that low ABPI is an individual risk factor for CAD due to its increased prevalence in CAD group.

\section{KEYWORDS}

Ankle Brachial Pressure Index, Coronary Arterial Disease.

HOW TO CITE THIS ARTICLE: Vengojayaprasad S, Shanmugam A, Bronson SC, et al. A comparative study of ankle-arm pressure index in coronary arterial disease patients and normal population - A South Indian tertiary care centre experience. J. Evolution Med. Dent. Sci. 2017;6(3):227-231, DOI: 10.14260/Jemds/2017/52

Financial or Other, Competing Interest: None.

Submission 04-12-2016, Peer Review 25-12-2016,

Acceptance 03-01-2017, Published 09-01-2017.

Corresponding Author:

Alagianambi Shanmugam,

Associate Professor,

Institute of Diabetology,

Government Stanley Medical College,

Chennai-01.

E-mail:drshanmugaa@gmail.com

DOI: $10.14260 /$ jemds $/ 2017 / 52$

\section{BACKGROUND}

American Heart Association Prevention Conference V described Ankle brachial pressure index (ABPI) test as a "simple, inexpensive, noninvasive measure" of peripheral arterial disease and stated that an abnormal ABI "provides incremental coronary and all cardiovascular disease risk assessment information". Measurement of ABPI has been recommended to improve cardiovascular risk assessment in individuals at "intermediate or higher risk of coronary arterial disease (CAD) on basis of traditional risk factor assessment".(1) Although several studies have demonstrated that ABPI $<0.9$ predicts future CAD mortality, there is little data regarding the utility of ABPI as a screening test in individuals for whom physicians actually order noninvasive test for cardiovascular risk prediction. 
So, this study was performed to find the prevalence of low ABPI $(<0.9)$ in clinical cases of coronary arterial disease patients and compare it with those who were free of disease clinically in the same group of population.

Low ABPI reflects peripheral arterial disease of lower limbs and is used as a tool in treating an ischaemic limb due to any cause, most common of which is atherosclerosis. Epidemiological studies have demonstrated that subclinical cardiovascular disease in one vascular bed is associated with the presence of clinical disease in another bed as well as with subsequent cardiovascular and total mortality. As coronary arterial disease also shares a common aetiology of atherosclerosis with peripheral arterial disease, ABPI can be used to predict its risk. Individuals with peripheral arterial disease of the lower extremities are among the highest-risk vascular patients. Therefore, presence of peripheral arterial disease is an indicator of widespread atherosclerosis in other vascular territories such as the coronary, carotid, and cerebrovascular arteries $(9, \mathrm{a}-\mathrm{d})$.

\section{MATERIALS AND METHODS}

ABPI was compared between 100 patients with clinical cardiovascular disease versus 100 controls taken from patients attending Coimbatore Medical College and general population of Coimbatore respectively. ABPI $<0.9$ was taken as cut-off, as it has a sensitivity of $90 \%$ in detecting peripheral vascular disease, which may also reflect similar pathology in other vascular bed, like coronary arteries. Prevalence of ABPI less than 0.9 was compared and analysed between the two study populations.

The ABPI was measured according to a standard protocol. Vascular lower limb examination was done by feeling the foot pulses (dorsalis pedis and posterior tibial arteries), and identifying the best palpable one. ABPI was measured by using the sphygmomanometer and a sonic aid hand-held Doppler probe (GIMA Ultrasound Technology Ltd, UK), utilising the best identified note of foot arteries and measuring its index in relation to the brachial artery. The ABI measurements were compared between the two groups, with special concentration on patients with hypertension, diabetes, hypertension, smoking, PAD, etc as comorbid associations of atherosclerosis.

Chi-square test was used to compare percentages. A p value of $\leq 0.05$ was considered statistically significant.

\section{Study Population \\ Patients}

100 (50 men and 50 women) randomly selected coronary arterial disease patients (as evidenced by prior medical records, recent biochemical, ECG, echocardiographic data, etc) attending Cardiac Department of Coimbatore Medical College were screened for ABPI.

\section{Controls}

Age and sex matched controls, 100 in number from general population, who showed no symptoms or signs of coronary arterial disease.

\section{Special Remarks}

Other confounders of CAD like hypertension, diabetes, dyslipidaemia, genetic predispositions, smokers, etc were not matched. These were taken as separate variables for statistical analysis.

\section{Exclusion Criteria}

Patients who were acutely ill or significantly morbid were excluded from participating in the study.

\section{RESULTS AND DISCUSSION}

\section{Relationship of Coronary Arterial Disease to ABPI}

Of the 100 patients with coronary arterial disease, $34 \%$ had an ABPI value below 0.9. ABPI in normal range of 0.9 to 1.2 was found in $58 \%$ of total. Another $8 \%$ had ABPI in higher range, which is usually associated with calcification of the vessel, which again is a risk for coronary arterial disease.

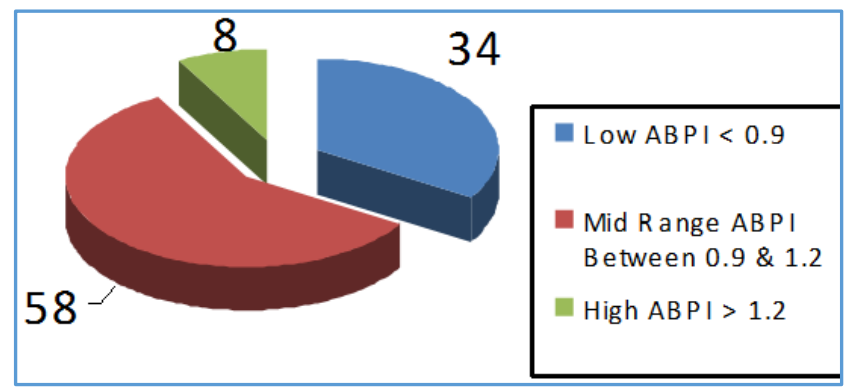

Figure 1. ABPI vs. CAD

The people in the control group had significantly lesser numbers falling in the abnormal range. $77 \%$ percentage of people had ABPI in the normal range. Only $11 \%$ of people had ABPI in the range of ischaemic limb, i.e. below 0.9. Though $12 \%$ had a higher ABPI value, it should be because of the prevalence of higher age and the comorbid conditions in the group.

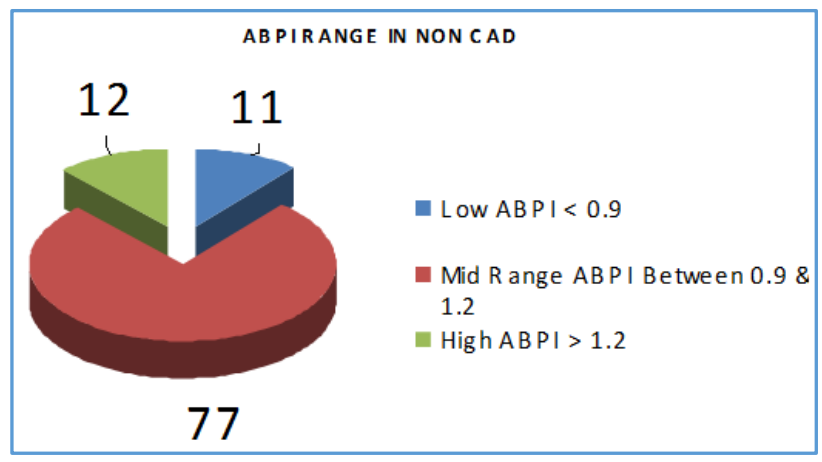

Figure 2. ABPI vs. non-CAD

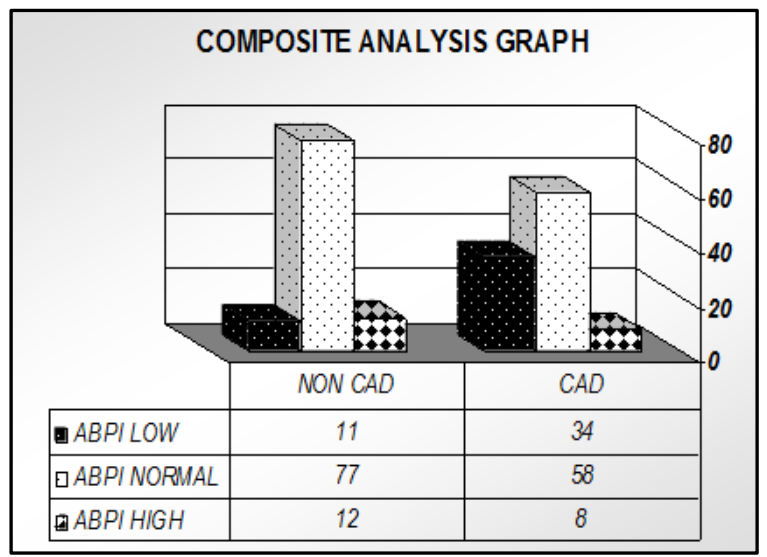

Figure 3. ABPI in CAD vs. non-CAD

Figure 3 shows the comparison of low ABPI in CAD vs. non-CAD group. There is a significant increase of low ABPI in CAD group. It was found that in student's t-test the value was statistically significant. The correlation coefficient was 0.239 
and Pearson coefficient was 0.239 . This further confirms that the null hypothesis (there is significant difference between ABPI levels in CAD and non-CAD groups) is true. ABPI level is high in non-CAD group as compared to CAD group. Non-CAD groups have normal ABPI value.

\section{Effect of Age on ABPI}

The effect of age on ABPI in both groups was studied. With higher age, more numbers were seen in the abnormal ABPI range. The trend line was plotted. It was seen that in the coronary arterial disease patients, people with lower age had abnormal ABPI readings compared to the normal patients.

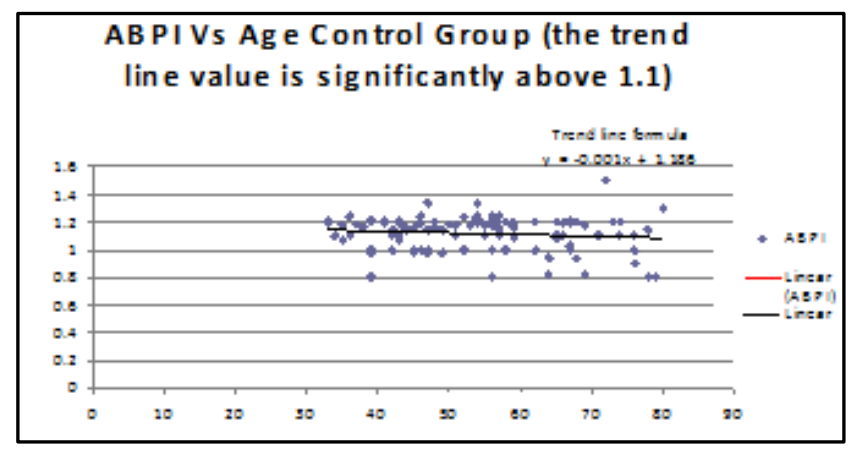

Figure 4 a. Age vs. ABPI (Control group)

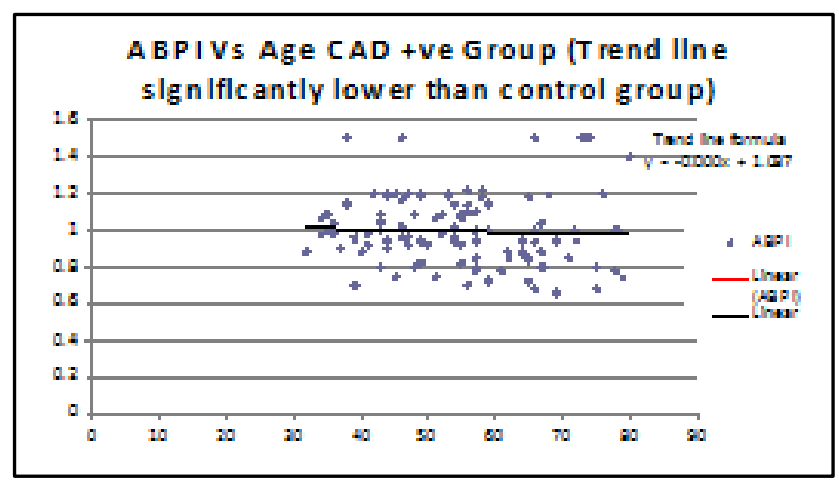

Figure 4b. Age vs. ABPI (CAD Group)

\section{Analysis of Other Comorbid Conditions in the Study} Relation of Hypertension with the Study Groups

80 people in the CAD group had hypertension as compared to 44 in non-CAD group (Figure $5 \mathrm{a}$ ). The prevalence of low ABPI in CAD group was increased when compared to non-CAD group. $33.75 \%$ in hypertensive CAD group had low ABPI as compared to $15.90 \%$ in the hypertensive non-CAD group.

Lowest prevalence was in the non-hypertensive, non-CAD group where the incidence was just $7 \%$.

The highest prevalence was noted in the hypertensive CAD group, where it formed $33.75 \%$ of the group, as seen in figure $5 b$.

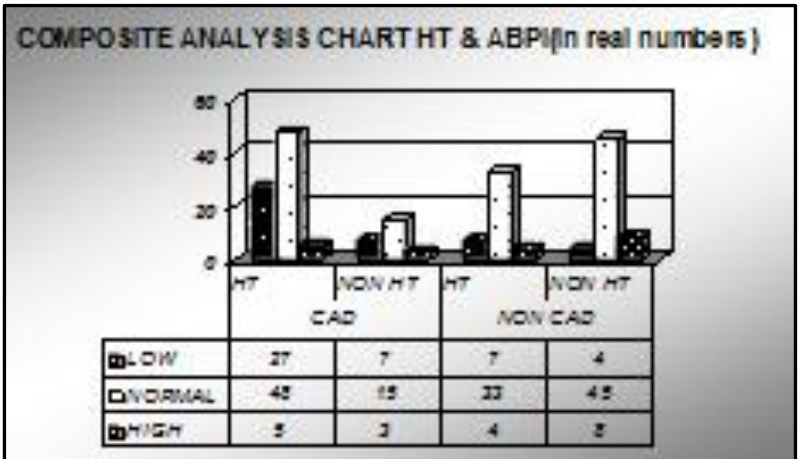

Figure 5a. Hypertensive Chart in Real Numbers

Figure 5a shows that the composite analysis chart of Hypertension and ABPI levels in real numbers. CAD patients with Hypertension had significantly low ABPI as compared to non-CAD patients with hypertension. In CAD with NonHypertension group and Non-CAD with Hypertension group the Low ABPI level is same.

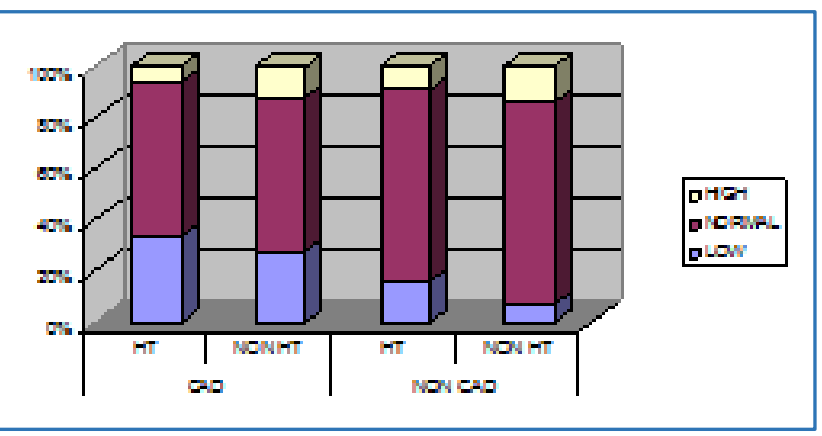

Figure 5b. Graph Plotted in Percentage Prevalence of ABPI range in Hypertensives In both Groups

Figure $5 \mathrm{~b}$ shows that the percentage prevalence of ABPI ranges in hypertensives in both $\mathrm{CAD}$ and non-CAD group. Non-CAD group with Non-Hypertension had lowest prevalence of ABPI. Low prevalence of ABPI is more in CAD with hypertension as compared to non-CAD with Hypertension. Non-CAD with Non-Hypertension group had normal ABPI prevalence. In CAD group with Hypertension and Non-Hypertension the low and normal ABPI prevalence are approximately same.

\section{Effect of Diabetes ABPI in the Study Group}

In the CAD group, $40 \%$ had diabetes.

In the non-CAD group, $38 \%$ had diabetes.

Highest prevalence of low ABPI was found in CAD with diabetes, constituting 40\% (Figure 7) of 60 (figure 6b). Lowest prevalence of low ABPI was found in people without both diabetes and CAD, constituting 11\% (Figure 7) of 62 (Figure 6a). 


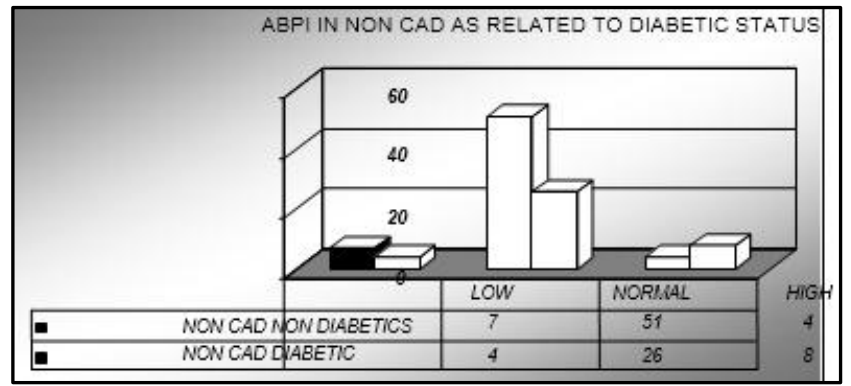

Figure 6a. ABPI in non-CAD as Related to Diabetic Status

Figure 6a shows the ABPI levels on Non-CAD + NonDiabetic to Non-CAD + Diabetic. High level of ABPI in NonCAD + Diabetic is twice than of Non-CAD + Non-Diabetic. NonCAD + Diabetic group had lower ABPI level. Non-CAD + NonDiabetic had more normal ABPI levels as compared to NonCAD + Diabetic group.

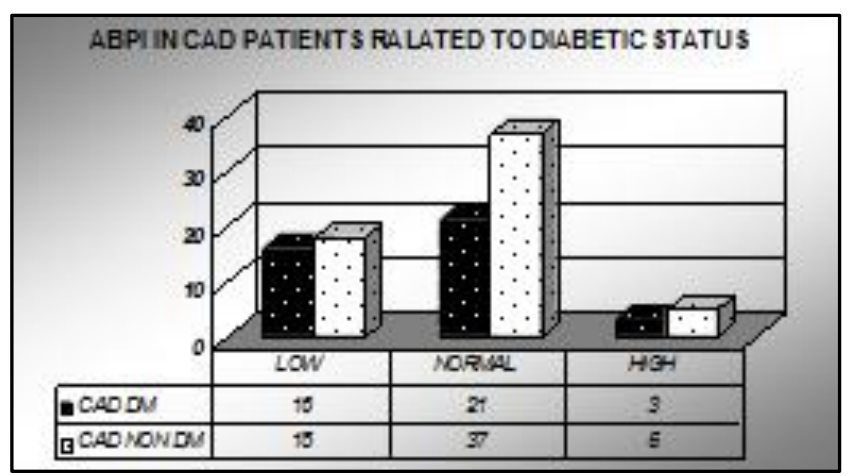

Figure 6b. ABPI in CAD Patients related to Diabetic Status

Figure $6 \mathrm{~b}$ shows that the ABPI in CAD + Diabetes patients and $\mathrm{CAD}+$ Non-Diabetes patients. There is significant difference in Low ABPI levels in CAD + Diabetes group and CAD + Non-Diabetes group. CAD + Non-Diabetes group had normal ABPI level. High level of ABPI is less in CAD + Diabetes group as compared to CAD + Non-Diabetes group.

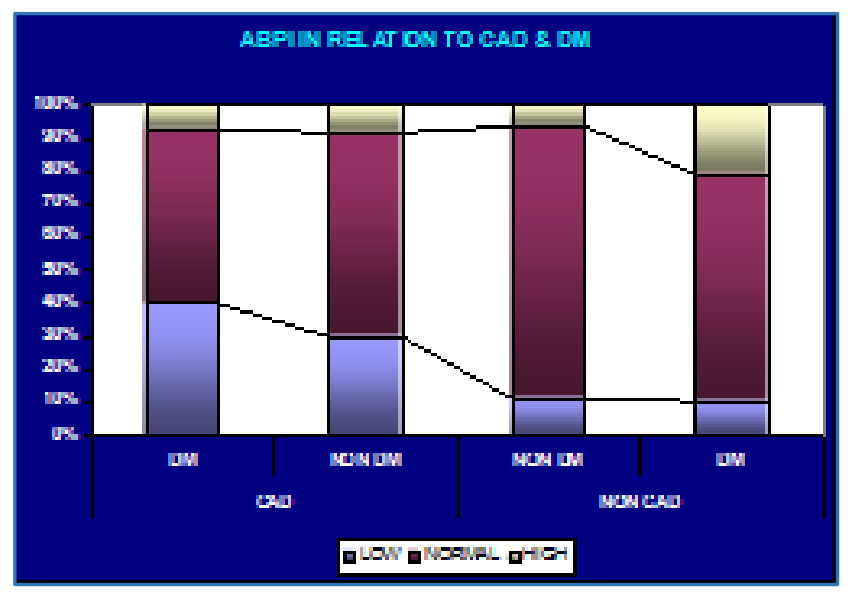

Figure 7. ABPI in Relation to CAD and DM in Percentage

Figure 7 shows that ABPI in relation to CAD and Non-CAD in both Diabetes and Non-Diabetes, Non-CAD + Diabetes group had low and High ABPI value. In CAD + Diabetes and Non-diabetes, the ABPI levels are approximately same. NonCAD + Non-diabetes had highest normal ABPI level.

\section{Effect of Dyslipidaemia on ABPI}

Prevalence of low ABPI in dyslipidaemic individuals in both groups was plotted. 46 of CAD group and 57 of the Non-CAD group had dyslipidaemia (Figure 8a). Of the dyslipidaemics, $33 \%$ of the CAD group and $35 \%$ of the Non-CAD group had low ABPI. Only $11 \%$ had low ABPI in the people who did not have both dyslipidaemia and CAD.

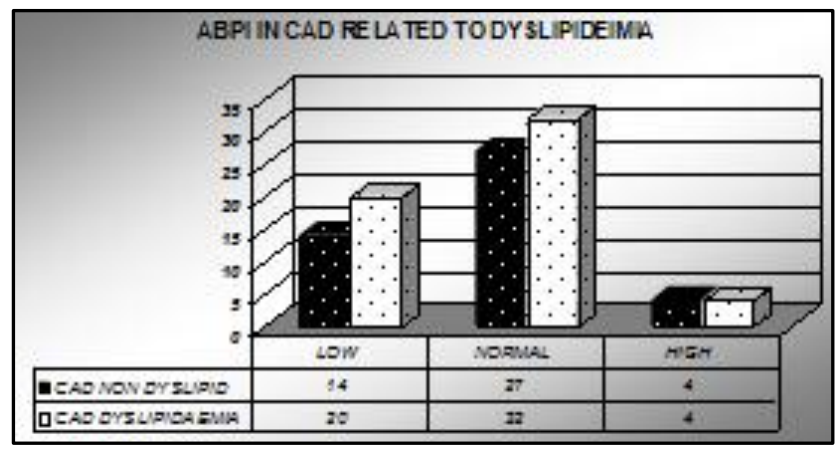

Figure 8a. ABPI in CAD related to Dyslipidaemia

Figure 8a shows the ABPI in CAD related to Dyslipidaemia. In both CAD + Non-Dyslipidaemia and CAD + Dyslipidaemia the High level of ABPI is same. CAD with NonDyslipidaemia had low level of ABPI than CAD + Dyslipidaemia. CAD + Dyslipidaemia groups had normal ABPI level.

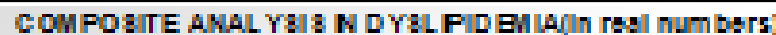

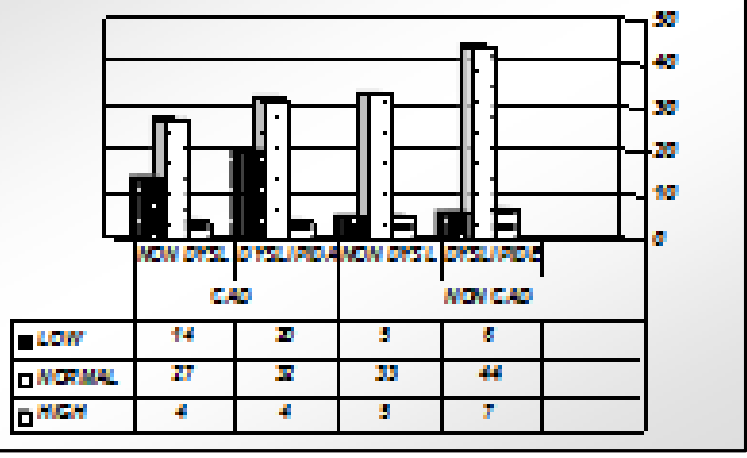

Figure 8b. Composite analysis in Dyslipidaemia

Figure $8 \mathrm{~b}$ shows the composite analysis of dyslipidaemia in CAD and Non-CAD groups. High level of ABPI is same in CAD + Non-Dyslipidaemia and Dyslipidaemia. Non-CAD + Non-Dyslipidaemia group had low ABPI level. In Non-CAD + Non-dyslipidaemic group, the low and high ABPI levels are same. Non-CAD + Non-Dyslipidaemia group had comparatively normal ABPI level.

\section{CONCLUSION}

- There is a definite increase in prevalence of low ABPI in patients with coronary arterial disease in this study.

- The study can be concluding by the remark that low ABPI is an individual risk factor for CAD, due to its increased prevalence in CAD group.

- Increasing age has a lowering effect on ABPI, both in normal as well as patients with coronary arterial disease.

- Diabetes has significant impact on ABPI in patients with CAD as observed in the study. It can be concluded that a 
diabetic has a definite risk of lowering ABPI as an isolated factor, which needs future study.

- Hypertension and Dyslipidaemia were also found to increase the prevalence of low ABPI in coronary arterial disease patients. Dyslipidaemia has a definite role in CAD patients and low ABPI is an index which has been observed.

\section{Acknowledgement}

Authors would like to thank all the participants of the study. Authors also acknowledge the immense help received from the scholars whose articles are cited and included in reference of this manuscript. The authors are also grateful to authors, editors and publishers of all those articles, journals and books from where the literature for this article has been reviewed and discussed.

\section{REFERENCES}

[1] McDermott MM, Liu, K, Guralnik, JM, et al. The ankle brachial index independently predicts walking velocity and walking endurance in peripheral arterial disease. J Am Geriatr Soc 1998;46(11):1355-62.

[2] Clement DL, Boccalon H, Dormandy J, et al. A clinical approach to the management of the patient with coronary (Co) and/or carotid (Ca) artery disease who presents with leg ischaemia (Lis). Int Angiol 2000;19(2):97-125.
[3] Criqui MH, Langer RD, Fronek A, et al. Mortality over a period of 10 years in patients with peripheral arterial disease. N Engl J Med 1992;326(6):381-6.

[4] Smith GD, Shipley MJ, Rose G. Intermittent claudication, heart disease risk factors, and mortality. The Whitehall study. Circulation 1990;82(6):1925-31.

[5] McKenna M, Wolfson S, Kuller L. The ratio of ankle and arm arterial pressure as an independent predictor of mortality. Atherosclerosis 1991;87(2-3):119-28.

[6] Newman AB, Siscovick DS, Manolio TA, et al. Ankle-arm index as a marker of atherosclerosis in the cardiovascular health study. Cardiovascular heart study (CHS) collaborative research group. Circulation 1993;88(3):837-45.

[7] Newman AB, Tyrrell KS, Kuller LH. Mortality over four years in SHEP participants with a low ankle-arm index. J Am Geriatr Soc 1997;45(12):1472-8.

[8] Newman AB, Shemanski L, Manolio TA, et al. Ankle-arm index as predictor of cardiovascular disease and mortality in the cardiovascular health study. The cardiovascular health study group. Arterioscler Thromb Vasc Biol 1999;19(3):538-45. 